POS $\quad$ PROCEEDINGS

\title{
Latest results from NA57
}

\section{Giuseppe Eugenio Bruno*}

University and INFN Bari, Italy

E-mail: giuseppe.bruno@ba.infn.it

for the NA57 collaboration: F Antinori ${ }^{k}, \mathbf{P ~ B a c o n}^{e}, \mathbf{A}$ Badalà $^{f}, \mathbf{R}$ Barbera $^{f}, \mathbf{A}$ Belogianni $^{a}$, I J Bloodworth ${ }^{e}$, M Bombara $^{h}$, G E Bruno ${ }^{b}$ S A Bull ${ }^{e}$, R Caliandro ${ }^{b}$, M Campbell ${ }^{g}$, W Carena ${ }^{g}$,

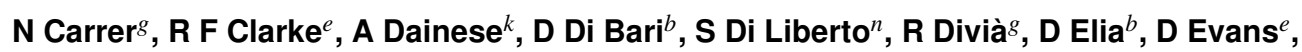

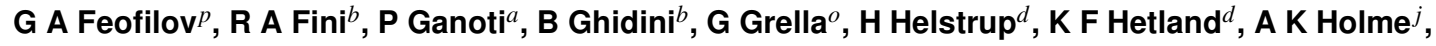
A Jacholkowski ${ }^{f}$, G T Jones ${ }^{e}, \mathbf{P}$ Jovanovic $^{e}, \mathbf{A ~ J u s k o}^{e}, \mathbf{R}$ Kamermans $^{r}, \mathbf{J}$ B Kinson ${ }^{e}, \mathbf{K} \mathrm{Knudson}^{g}$, V Kondratiev ${ }^{p}$, I Králik ${ }^{h}, \mathbf{A}$ Kravčáková $^{i}, \mathbf{P}$ Kuijer $^{r}, \mathbf{V}$ Lenti $^{b}, \mathbf{R}$ Lietava $^{e}, \mathbf{G ~ L ø v h ø i d e n ~}^{j}, \mathbf{V ~ M a n z a r i ~}^{b}$,

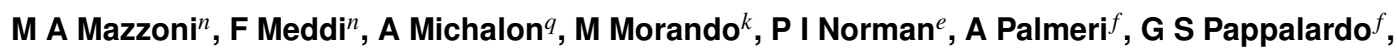
B Pastirčák ${ }^{h}, \mathbf{R}$ J Platt ${ }^{e}, \mathbf{E}$ Quercigh $^{k}, \mathbf{F}$ Riggi $^{f}, \mathbf{D}$ Röhrich $^{c}, \mathbf{G}$ Romano $^{o}, \mathbf{K}$ Šafařík $^{g}, \mathbf{L ~ S ̌ a ́ n d o r ~}^{h}$, E Schillings $^{r}$, G Segato ${ }^{k}$, M Sené $^{l}$, R Sené ${ }^{\prime}$, W Snoeys ${ }^{g}$, F Soramel $^{k}$ M Spyropoulou-Stassinaki $^{a}$, $\mathbf{P ~ S t a r o b a}^{m}, \mathbf{R}$ Turrisi $^{k}, \mathbf{T} \mathbf{S}$ Tveter $^{j}, \mathbf{J ~ U r b a ́ n ~}^{i}, \mathbf{P}$ van de Ven ${ }^{r}, \mathbf{P}$ Vande Vyvre ${ }^{g}, \mathbf{A}$ Vascotto $^{g}, \mathbf{T ~ V i k}^{j}$, O Villalobos Baillie ${ }^{e}, \mathbf{L}$ Vinogradov ${ }^{p}, \mathbf{T}$ Virgili $^{o}, \mathbf{M} \mathbf{F}$ Votruba $^{e}, \mathbf{J}$ Vrláková $^{i}$ and $\mathbf{P}$ Závada $^{m}$

a Physics Department, University of Athens, Athens, Greece

${ }^{b}$ Dipartimento IA di Fisica dell'Università e del Politecnico di Bari and INFN, Bari, Italy

${ }^{c}$ Fysisk Institutt, Universitetet i Bergen, Bergen, Norway

${ }^{d}$ Høgskolen i Bergen, Bergen, Norway

${ }^{e}$ University of Birmingham, Birmingham, $U K$

${ }^{f}$ University of Catania and INFN, Catania, Italy

${ }^{g}$ CERN, European Laboratory for Particle Physics, Geneva, Switzerland

${ }^{h}$ Institute of Experimental Physics, Slovak Academy of Science, Košice, Slovakia

${ }^{i}$ P.J. Šafárik University, Košice, Slovakia

${ }^{j}$ Fysisk Institutt, Universitetet i Oslo, Oslo, Norway

${ }^{k}$ University of Padua and INFN, Padua, Italy

${ }^{l}$ Collège de France, Paris, France

${ }^{m}$ Institute of Physics, Prague, Czech Republic

${ }^{n}$ University "La Sapienza" and INFN, Rome, Italy

o Dipartimento di Scienze Fisiche "E.R. Caianiello" dell'Università and INFN, Salerno, Italy

${ }^{p}$ State University of St. Petersburg, St. Petersburg, Russia

${ }^{q}$ IReS/ULP, Strasbourg, France

${ }^{r}$ Utrecht University and NIKHEF, Utrecht, The Netherlands

NA57 at the CERN SPS has studied the production of strange particles in $\mathrm{Pb}-\mathrm{Pb}$ and $\mathrm{p}-\mathrm{Be}$ collisions. Hyperon enhancements at $40 \mathrm{~A} \mathrm{GeV/c}$ are presented and compared to results at 160 $\mathrm{GeV} / c$ beam momenta. The momentum spectra are analysed based on hydrodynamical models and freeze-out temperature, transverse and longitudinal flow velocities are extracted. Central-toperipheral nuclear modification factors at $160 \mathrm{~A} \mathrm{GeV/c}$ are calculated.

International Europhysics Conference on High Energy Physics

July 21st - 27th 2005

Lisboa, Portugal

\footnotetext{
${ }^{*}$ Speaker.
} 


\section{Strangeness Enhancement}

An enhanced production of strange particles in nucleus-nucleus collisions with respect to proton-induced reactions was suggested long ago as a possible signature of the phase transition from colour-confined hadronic matter to a Quark-Gluon-Plasma (QGP) [1]. NA57 has measured the production of $\Lambda, \Xi^{-}, \Omega^{-}$, their antiparticles and $\mathrm{K}_{\mathrm{S}}^{0}$ in $\mathrm{Pb}-\mathrm{Pb}$ and in reference collisions (p$\mathrm{Be}$ and $\mathrm{p}-\mathrm{Pb})$ at 40 and $160 \mathrm{~A} \mathrm{GeV} / \mathrm{c}$ in the central unit of rapidity and at medium transverse momentum $\left(p_{\mathrm{T}} \gtrsim 0.5 \mathrm{GeV} / c\right)[2,3]$. The Enhancement $E$ is defined as the yield per participant $\left(Y /<N_{\text {wound }}>, Y=\int_{y_{c m-0.5}}^{y_{c m+5}} \mathrm{~d} y \int_{0}^{\infty} \frac{\mathrm{d} N^{2}}{\mathrm{~d} p_{\mathrm{T}} \mathrm{d} y} \mathrm{~d} p_{\mathrm{T}}\right)$ relative to $\mathrm{p}$-Be collisions. The hyperon enhancements at 40 and $160 \mathrm{~A} \mathrm{GeV/c}$ are shown as a function of centrality in fig. 1 . The arrow in the $2^{\text {nd }}$ panel of fig. 1 indicates the lower limit to the $\bar{\Xi}^{+}$enhancement in the four most central classes at 95\% confidence level. At $40 \mathrm{~A} \mathrm{GeV} / \mathrm{c}$ the enhancement pattern follows the same hierarchy with the strangeness content predicted in a QGP scenario: $E(\Lambda)<E\left(\Xi^{-}\right)$and $E(\bar{\Lambda})<E\left(\bar{\Xi}^{+}\right)$, as already observed at $160 \mathrm{~A} \mathrm{GeV/c}$ where further $E(\Xi)<E(\Omega)$ [3]. The rational behind these predictions is that the $s$ and $\bar{s}$ quarks, abundantly produced in the deconfined phase would recombine to form strange and multi-strange particles in a time much shorter than that required to produce them by successive rescattering interactions in a hadronic gas. Comparing the measurements at the two
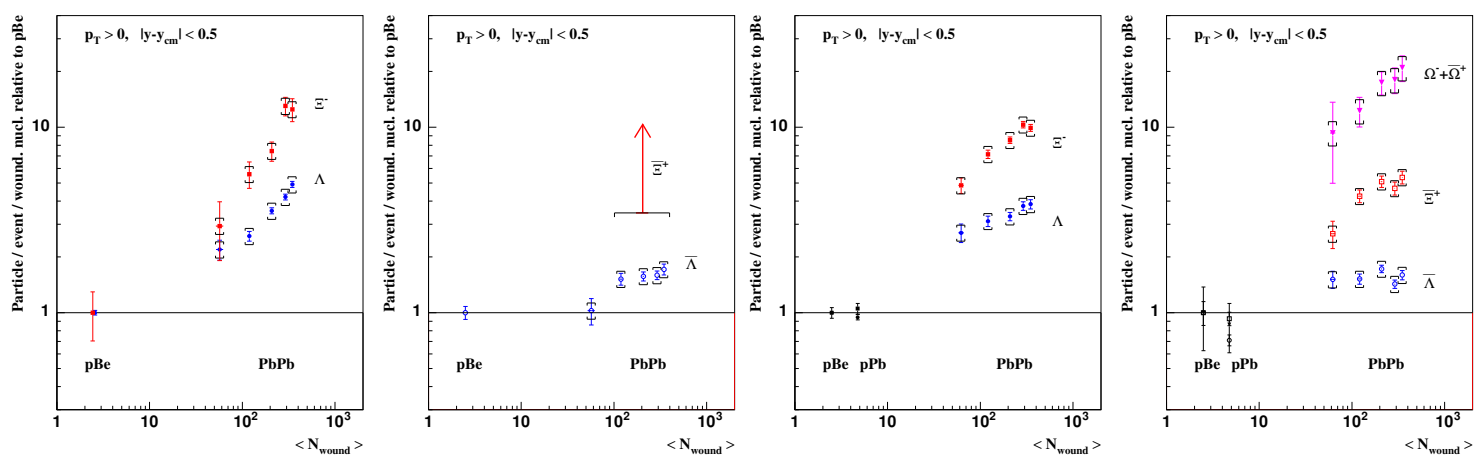

Figure 1: Hyperon enhancements as a function of the number of participants $\left(N_{\text {wound }}\right)$ at $40\left(1^{\text {st }}\right.$ and $2^{\text {nd }}$ panels) and 160 ( $3^{r d}$ and $4^{\text {th }}$ panels) $A \mathrm{GeV} / c$. The symbol $\square$ shows the systematic error.

beam momenta: for the most central collisions the enhancements are slightly larger at 40 than at $160 \mathrm{GeV} / c$, the increase with $N_{\text {wound }}$ is steeper at 40 than at $160 \mathrm{GeV} / c$.

\section{Collective Dynamics}

The expansion dynamics of the $\mathrm{Pb}-\mathrm{Pb}$ collisions has been studied in the transverse and longitudinal directions by measuring, respectively, the $m_{\mathrm{T}}\left(=\sqrt{p_{\mathrm{T}}^{2}+m^{2}}\right)$ spectra (details in ref. [5, 6]) and the rapidity distributions (details in ref. [7]) of strange particles, based on a hydro-dynamics inspired model [8]. A simultaneous description of all the measured particle spectra, both for rapidity and $p_{\mathrm{T}}$ can be achieved with the following set of parameters (for the most central $53 \%$ of the inelastic $\mathrm{Pb}-\mathrm{Pb}$ cross section at $160 \mathrm{~A} \mathrm{GeV} / \mathrm{c}$ ): freeze-out temperature $T=144 \pm 7 \mathrm{MeV}$, average flow velocities $\left\langle\beta_{\perp}\right\rangle=0.38 \pm 0.02$ and $\left\langle\beta_{\|}\right\rangle=0.42 \pm 0.03$, as shown in fig. 2 . With increasing centrality, the freeze-out temperature decreases and the transverse flow velocity increases. In the 

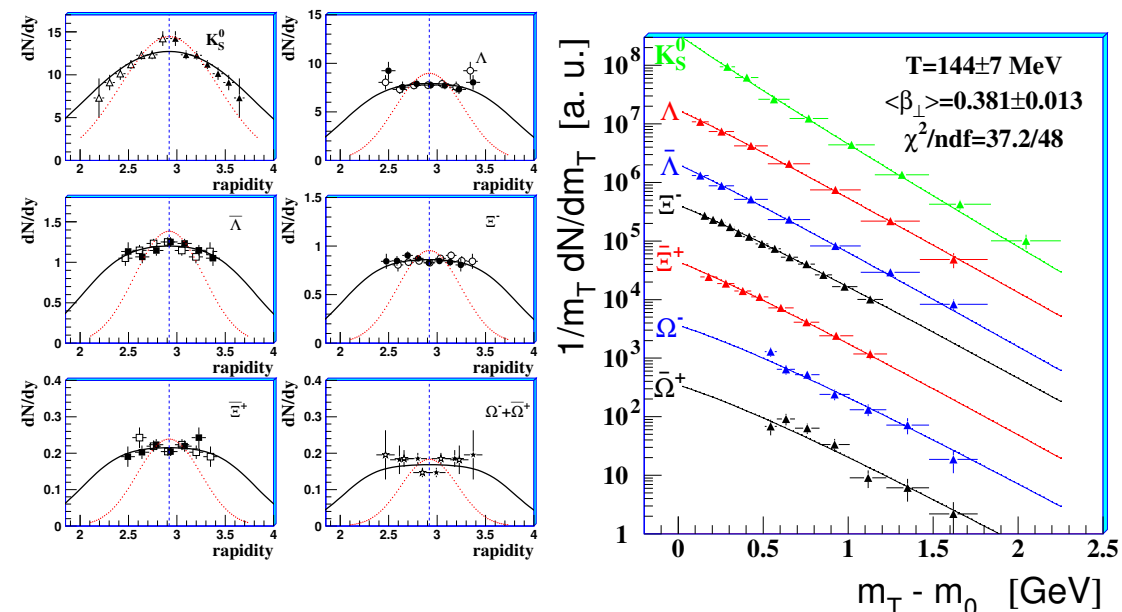

Figure 2: Rapidity distributions (left) and transverse mass spectra (right) of strange particles for the most central $53 \%$ of the inelastic $\mathrm{Pb}-\mathrm{Pb}$ cross-section. Blast-wave fits are superimposed to the data point (full lines). The dotted lines in the left panel show the distribution expected for a thermal source without any longitudinal flow.

longitudinal direction, instead, we do not observe a centrality dependence. The freeze-out temperature is lower at $40(T=118 \pm 5 \mathrm{MeV})$ than at $160 \mathrm{~A} \mathrm{GeV} / c$, but the transverse velocity is compatible at the two energies.

\section{Nuclear Modification Factors}

At the Relativistic Heavy Ion Collider (RHIC), the central-to-peripheral nuclear modification factor

$$
R_{\mathrm{CP}}\left(p_{\mathrm{T}}\right)=\frac{\left\langle N_{\text {coll }}\right\rangle_{\mathrm{P}}}{\left\langle N_{\text {coll }}\right\rangle_{\mathrm{C}}} \times \frac{\mathrm{d}^{2} N_{\mathrm{AA}}^{\mathrm{C}} / \mathrm{d} p_{\mathrm{T}} \mathrm{d} y}{\mathrm{~d}^{2} N_{\mathrm{AA}}^{\mathrm{P}} / \mathrm{d} p_{\mathrm{T}} \mathrm{d} y}
$$

measured for a large variety of particles has proven to be a powerful tool for the study of parton propagation in the dense QCD medium expected to be formed in nucleus-nucleus collisions (see, e.g., [9]). At SPS energy, so far, only $\pi^{0} R_{\mathrm{CP}}$ measurements were available [10]; the first results on the particle-species dependence (unidentified negatively charged hadrons, $\mathrm{K}_{\mathrm{S}}^{0}, \Lambda$, and $\bar{\Lambda}$ ) were reported recently by the NA57 Collaboration in [11]. Figure 3 (left panel) shows the results for $0-5 \% / 40-55 \% R_{\mathrm{CP}}$ nuclear modification factors. At low- $p_{\mathrm{T}} R_{\mathrm{CP}}$ scales with the number of participants for all particles except the $\bar{\Lambda}$. With increasing $p_{\mathrm{T}}, \mathrm{K}_{\mathrm{S}}^{0}$ mesons reach values of $R_{\mathrm{CP}} \approx 1$ : we do not observe the enhancement above unity that was measured in proton-nucleus relative to pp collisions (Cronin effect [12]). An enhancement is, instead, observed for strange baryons, $\Lambda$ and $\bar{\Lambda}$, that reach $R_{\mathrm{CP}} \simeq 1.5$ at $p_{\mathrm{T}} \simeq 3 \mathrm{GeV} / c$. In fig. 3 (middle panel) we compare our $\mathrm{K}_{\mathrm{S}}^{0}$ data to predictions (X.N. Wang) obtained from a perturbative-QCD-based calculation [13], including (thick line) or excluding (thin line) in-medium parton energy loss. The initial gluon rapidity density of the medium was scaled down, from that needed to describe RHIC data, according to the decrease by about a factor 2 in the charged particle multiplicity. The data are better described by the curve that does include energy loss. The prediction of a second model of parton energy loss (PQM) that describes several energy-loss-related observables at RHIC energies [14] is also in agreement with the value reached at high- $p_{\mathrm{T}}$ by the data. Figure 3 (right panel) shows the ratio of 

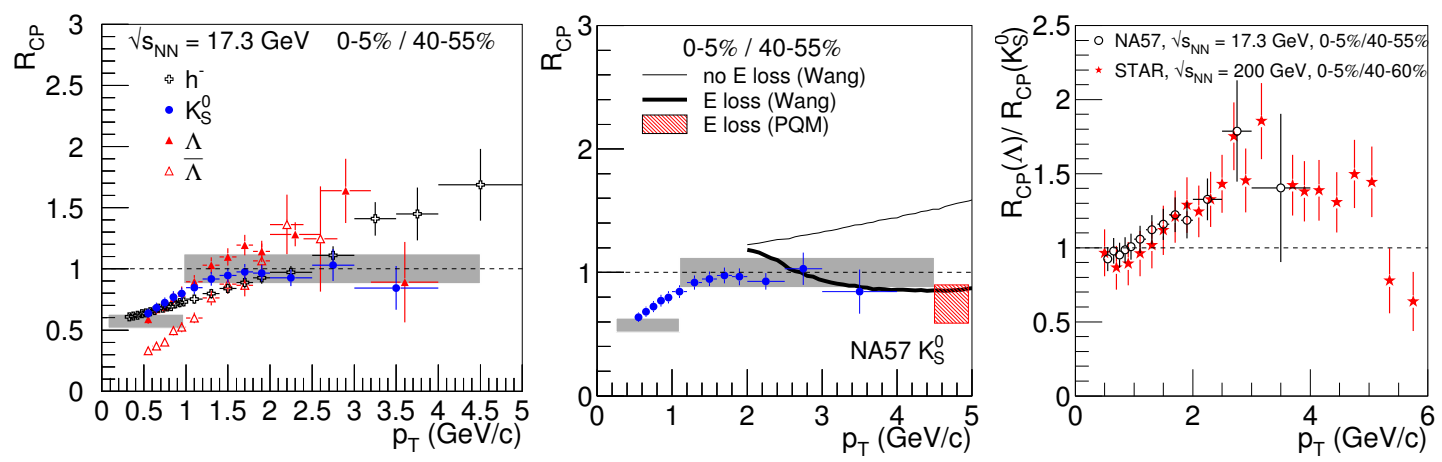

Figure 3: Left: $R_{\mathrm{CP}}$ ratios for negatively charged particles $\left(h^{-}\right)$and singly-strange particles in $\mathrm{Pb}-\mathrm{Pb}$ collisions at $\sqrt{s_{\mathrm{NN}}}=17.3 \mathrm{GeV}$ [11]. The width of the shaded band centered at $R_{\mathrm{CP}}=1$ indicates the systematic error due to the uncertainty on the values of $\left\langle N_{\text {coll }}\right\rangle$ in each class; the band at low $p_{\mathrm{T}}$ show the value expected for scaling with the number of participants. Middle: $\mathrm{K}_{\mathrm{S}}^{0} R_{\mathrm{CP}}\left(p_{\mathrm{T}}\right)$ compared to predictions $[13,14]$ with and without energy loss Right ratio of $\Lambda R_{\mathrm{CP}}$ to $\mathrm{K}_{\mathrm{S}}^{0} R_{\mathrm{CP}}$, as a function of $p_{\mathrm{T}}$, at the SPS (NA57 at $\left.\sqrt{s_{\mathrm{NN}}}=17.3 \mathrm{GeV}\right)$ and at RHIC (STAR at $\left.\sqrt{s_{\mathrm{NN}}}=200 \mathrm{GeV}[9,15]\right)$.

$\Lambda R_{\mathrm{CP}}$ to $\mathrm{K}_{\mathrm{S}}^{0} R_{\mathrm{CP}}$, as measured from our data and by STAR at $\sqrt{s_{\mathrm{NN}}}=200 \mathrm{GeV}[9,15]$ (note that $\Lambda+\bar{\Lambda}$ are considered by STAR and that the centrality range used for the peripheral class is slightly different in the two experiments). The similarity of the $\Lambda-\mathrm{K}$ pattern to that observed at RHIC may be taken as an indication for coalescence effects [16] at SPS energy.

\section{References}

[1] Rafelski J and Müller B 1982 Phys. Rev. Lett. 48 1066, ibidem 1986562334

[2] Antinori F et al. 2004 Phis. Lett. B 595 68-74

[3] Bruno G E et al. 2004 J. Phys. G: Nucl. Part. Phys. 30 S717-S724

[4] Tounsi A, Mischke A and Redlich K 2003 Nucl. Phys. A 715 565c

[5] Antinori F et al. 2004 J. Phys. G: Nucl. Part. Phys. 30 823-840

[6] Bruno G E et. al. 2005 J. Phys. G: Nucl. Part. Phys. 31 S127-S133

[7] Antinori F et al. 2005 J. Phys. G: Nucl. Part. Phys. 31 1345-1357

[8] Schnedermann E, Sollfrank J and Heinz U 1993 Phys. Rev. C 48 2462-2475

[9] Adams J et al. 2004 Phys. Rev. Lett. 92052302

[10] Aggarwal M M et al. 2002 Eur. Phys. J. C 23 225; d'Enterria D 2004 Phys. Lett. B 59632.

[11] Antinori F et al. 2005 Phys. Lett. B 62317

[12] Cronin J et al. 1975 Phys. Rev. D 113105.

[13] Wang X N 2000 Phys. Rev. C C61 064910; private communication.

[14] A. Dainese et al., Eur. Phys. J. C38 (2005) 461; private communication.

[15] M.A.C. Lamont, poster presentation at QM2005, Acta Phys. Slov. in print.

[16] Hwa R C and Yang C B 2003 Phys. Rev. C 67 064902; Fries R J et al. 2003 Phys. Rev. C 68 044902; Greco V et al. Phys. Rev. C 68034904. 